\title{
Photosensitivity and hyperpigmentation secondary to amiodarone: single case report
}

\begin{abstract}
We report and emblematic case of a 65years-old male patient with cutaneous photosensitivity and progressive hyperpigmentation of photo exposed skin after continuous use of amiodarone to treat an atrial fibrillation developed along with chronic obstructive pulmonary disease. Relevant literature was consulted and is discussed together with the findings of the case.
\end{abstract}

Keywords: amiodarone, dermatology, photosensitivity, phototoxic reactions, hyperpigmentation
Volume 3 Issue 2 - 2019

\author{
Gabriela Mantovani Pazian,' Paula Graziela \\ Wessler,' Gabriel Amorim, ',2 Roberto \\ Moreira Amorim Filho, ${ }^{1,3}$ Gustavo Moreira \\ Amorim ${ }^{1,4}$ \\ 'Faculty of Medicine, South Santa Catarina University (UNISUL), \\ Brazil \\ 2Professor Ruben David Azulay Dermatology Institute, Santa \\ Casa da Misericórdia do Rio de Janeiro, Brazil \\ ${ }^{3}$ Private Dermatology Clinic, Brazil \\ ${ }^{4}$ Pathology Post-Graduation Program - Faculty of Medicine, \\ Federal University of Rio de Janeiro (UFRJ), Brazil
}

\author{
Correspondence: Gustavo Moreira Amorim, Faculty of \\ Medicine, South Santa Catarina University (UNISUL), Brazil, Tel \\ +55489998 27097 \\ Email gustavomoreiraamorim@hotmail.com
}

Received: February 19, 2018 | Published: March 06, 2018

\section{Introduction}

Amiodarone is an iodine-rich antiarrhythmic agent that is widely used in the control of ventricular and supraventricular arrhythmia, and is superior to other antiarrhythmic drugs for maintenance of sinus rhythm. ${ }^{1,2}$ It can be used both in the acute treatment of sustained ventricular tachyarrhythmia's regardless of hemodynamic stability, as in the chronic treatment of patients with left ventricular dysfunction and heart failure; it also serves as prophylaxis in the perioperative period of cardiac surgery and as adjuvant of the implantable cardioversor-defibrillator to reduce the number of shocks. ${ }^{3,4}$

Amiodarone can cause a large range of side effects due to toxicity to various organs and systems when used for long periods, particularly in high doses. The possibility of thyroid alterations, besides ocular, pulmonary, hepatic and cutaneous toxicities are highlighted. ${ }^{3,5}$ The most common side effects are bradycardia, hypotension, visual acuity disorders, nausea, intestinal constipation, phlebitis and thyroid changes. ${ }^{6,7}$

The incidence of cutaneous side effects is high and reaches $75 \%$ among patients in chronic and prolonged use of amiodarone. The most common are photoallergic and phototoxic reactions in addition to hyperpigmentation. Less commonly, urticaria, pruritus, erythema nodosum, purpura and erythema multiforme may occur. Rare effects are described as: Stevens Johnson syndrome and toxic epidermal necrolysis, IgA bullous dermatosis and pseudoporphyria. Some reports mention the potential carcinogenic effect of amiodarone, such as the appearance of basal cell carcinoma at sites of previous photosensitivity/phototoxicity. ${ }^{8-11}$ We report a case of a male patient with atrial fibrillation who presented a classic and exuberant reaction of photosensitivity and hyperpigmentation, discussing these findings with what is reported in the literature.

\section{Case report}

A 65-years old male patient, former smoker with a chronic obstructive pulmonary disease (COPD), during a hospitalization for acute lower respiratory tract infection evolved with tachycardia, palpitation, chest pain and respiratory failure. Electrocardiogram showed atrial fibrillation with rapid ventricular response. There was no hemodynamic instability. Patient was treated with chemical cardioversion with intravenous amiodarone, in addition to broadspectrum antibiotics and therapeutic measures for COPD.

After complete improvement, patient was discharged receiving as a continuous prescription, daily dose of amiodarone. Approximately 2 to 3 months after hospitalization, patient complained of increased skin sensitivity when exposed to the. He reported erythema and burning sensation of the skin even after short periods of sun exposure. The lesions cleared quickly with the use of moisturizer. He had an initial evaluation, having been medicated only with sunscreen. As the time went by, photosensitivity was maintained, and even intensified, and the lesions did not clear any more, but remained with residual hyperchromia.

One year after hospitalization, he was referred to out clinic. Dermatological exam showed an intense grayish brown hyperpigmentation of the skin over photo exposed areas, denoting clear photosensitivity. Covered areas were spared (Figures 1). Patient has been using amiodarone ever since. With the hypothesis of an amiodarone cutaneous side effect, an evaluation with the cardiologist was requested. It was recommended the suspension of the amiodarone and initiation of propafenone. In a recent consult with 3 months follow up after suspension, patient notice a reduction of photosensitivity symptoms and slight lightening of hyperchromia.

\section{Discussion}

The photosensitivity caused by amiodarone ranges from an increased capacity to tan to a burning sensation or itching at sites exposed to radiation, often accompanied by erythema. ${ }^{10}$ Clinical features of the photosensitivity response represent a phototoxic reaction to amiodarone and its major metabolite, mono-Ndesethylamiodarone. ${ }^{11}$ Approximately $50 \%$ of all amiodarone-treated patients develop symptoms of acute photosensitivity, usually after 
4months of continuous treatment and a minimal dose of $40 \mathrm{~g} .{ }^{11-14}$ Photosensitivity gradually declines and returns to normal 4 to 12 months after discontinuation of amiodarone therapy12. However, it can sometimes last several years after drug withdrawal. ${ }^{10}$

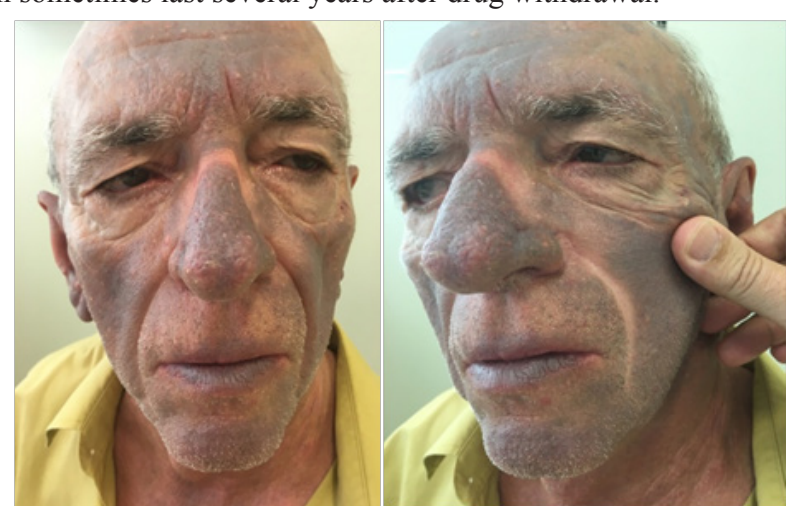

(A)

(B)

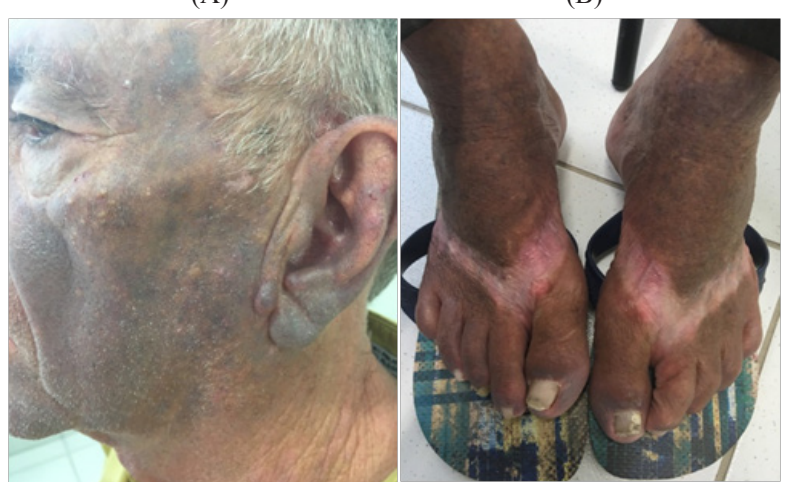

(C)

(D)

Figure I Dermatological exam showed an intense grayish brown hyperpigmentation of the skin over photo exposed areas, denoting clear photosensitivity. Covered areas were spared.

Amiodarone-related hyperpigmentation occurs due to skin drug deposition. Study conducted by Ammoury et al. ${ }^{11}$ published in 2008, identified the presence of high iodine concentrations, which was observed in the electronic probe analysis, suggesting that cutaneous deposits are composed of amiodarone itself or a metabolite. Therefore amiodarone deposits in the skin, with or without lipofuscin, are capable of inducing gray-blue hyperpigmentation. Cutaneous hyperpigmentation develops mainly in patients with Fitzpatrick's skin type, with an incidence of approximately $10 \%$ of treated patients. ${ }^{11}$ It occurs after an average of 20 months of continuous use and a minimal cumulative dose of $160 \mathrm{~g} .{ }^{12}$ The main treatment of both photosensitivity and hyperpigmentation is amiodarone suspension, in cases where another antiarrhythmic agent can replace its use. When it's not possible, dosage should be reduced to the minimum necessary to control cardiologic comorbidity, reducing deposition and contributing to faster tissue clearance..$^{15}$ Additionally it should be recommended daily use of broad spectrum sunscreen, preferably with zinc oxidecontaining preparations and avoid sun exposure. ${ }^{14}$ Other therapeutic options include chemical peels, narrow band UVB, Q-switched ruby laser. ${ }^{16,17}$

\section{Conclusion}

The authors present this emblematic case with good photographic documentation in order to make a point of the possible adverse effects inherent to amiodarone, a widely used antiarrhythmic drug. Adverse cutaneous manifestations related to its prolonged use are common and should be known by general practitioners. Although rarely serious, they determine an important impairment of patients' quality of life. Once identified and correctly diagnosed, suspension of the drug allows the improvement of the picture in most cases. However, the discontinuation of amiodarone should be discussed in a multidisciplinary team, considering its risks and benefits.

\section{Acknowledgments}

None.

\section{Conflicts of interest}

Authors declare that there is no conflict of interest.

\section{References}

1. Singh BN, Singh SN, DJ Reda, et al. Amiodarone versus sotalol for atrial fibrillation. $N$ Engl J Med. 2005;352(18):1861-1872.

2. Paiva E. Qual o papel da amiodarona no tratamento da fibrilação ventricular/taquicardia ventricular sem pulso? Rev Assoc Med Bras. 2001;47(2):100-111.

3. Vassallo P, Trohman RG. Prescribing amiodarone: an evidence-based review of clinical indications. JAMA. 2007;298(11):1312-1322.

4. Chevalier P, Durand-Dubief A, Burri H, et al. Amiodarone versus placebo and class Ic drugs for cardioversion of recent-onset atrial fibrillation: A meta-analysis. J Am Coll Cardiol. 2003;41(2):255-262.

5. Inaba H, Suzuki S, Takeda T, et al. Amiodarone-Induced Thyrotoxicosis with Thyroid Papillary Cancer in Multinodular Goiter: Case Report. Med Princ Pract. 2012;21(2):190-192.

6. Magalhães LP, Figueiredo MJO, et al. II Diretriz de fibrilação atrial. Arq Bras Cardiol. 2016;106(4 Supl.2):1-22.

7. Punnam SR, Goyal SK, Kotaru VP, et al. Amiodarone-a 'broad spectrum' antiarrhythmic drug. Cardiovasc Hematol Disord Drug Targets. 2010;10(1):73-81.

8. Jaworski K, Walecka I, Rudnicka L, et al. Cutaneous Adverse Reactions of Amiodarone. Med Sci Monit. 2014;20:2369-2372.

9. Blackshear JL, Randle HW. Reversibility of blue-gray cutaneous discoloration from amiodarone. Mayo Clin Proc. 1991;66(7):721-726.

10. Yones SS, O’Donoghue NB, Palmer RA, et al. Persistent severe amiodarone- induced photosensitivity. Clin Exp Dermatol. 2005;30(5):500-502.

11. Ammoury A, Michaud S, Paul C, et al. Photodistribution of bluegray hyper- pigmentation after amiodarone treatment: molecular characterization of amiodarone in the skin. Arch Dermatol. 2008;144(1):92-96.

12. Rappersberger K, Honigsmann H, Ortel B, et al. Photosensitivity and hyperpigmentation in amiodarone-treated patients: incidence, time course, and recovery. J Invest Dermatol. 1989;93(2):201-209.

13. Miller RA, McDonald AT. Dermal lipofuscinosis associated with amiodarone therapy: Report of a case. Arch Dermatol. 1984;120(5): 646-649.

14. Ferguson J, Addo HA, Jones S, et al. Amiodarone: a study of cutaneous photosensitivity. Br J Dermatol. 1985;113(5):537-539.

15. Drucker AM, Rosen CF. Drug-induced photosensitivity: culprit drugs, management and prevention. Drug Saf. 2011;34(10):821-837. 
16. Collins P, Ferguson J. Narrow-band UVB (TL-01) phototherapy: an effective preventative treatment for the photodermatoses. Br J Dermatol. $1995 ; 132(6): 956-963$.
17. Karrer S, Hohenleutner U, Szemies RM, et al. Amiodarone-induced pigmentation resolves after treatment with the Q-switched ruby laser. Arch Dermatol. 1999;135(3):251-253. 